\title{
Nutritional supplementation alters associations between one-carbon metabolites and cardiometabolic risk profiles in older adults: a secondary analysis of the Vienna Active Ageing Study
}

\author{
Nicola A. Gillies ${ }^{1,2} \cdot$ Bernhard Franzke $^{3} \cdot$ Barbara Wessner $^{3,4} \cdot$ Barbara Schober-Halper $^{3} \cdot$ Marlene Hofmann $^{3}$. \\ Stefan Oesen ${ }^{3} \cdot$ Anela Tosevska $^{3,5} \cdot$ Eva-Maria Strasser $^{6} \cdot$ Nicole C. Roy $^{1,2,7,8,9} \cdot$ Amber M. Milan $^{1,7,8}$. \\ David Cameron-Smith ${ }^{1,2,10} \cdot$ Karl-Heinz Wagner ${ }^{3,11}$ (1)
}

Received: 5 January 2021 / Accepted: 2 June 2021 / Published online: 8 July 2021

(c) The Author(s) 2021

\begin{abstract}
Purpose Cardiovascular diseases and cognitive decline, predominant in ageing populations, share common features of dysregulated one-carbon (1C) and cardiometabolic homeostasis. However, few studies have addressed the impact of multifaceted lifestyle interventions in older adults that combine both nutritional supplementation and resistance training on the co-regulation of $1 \mathrm{C}$ metabolites and cardiometabolic markers.

Methods 95 institutionalised older adults ( $83 \pm 6$ years, $88.4 \%$ female) were randomised to receive resistance training with or without nutritional supplementation (Fortifit), or cognitive training (control for socialisation) for 6 months. Fasting plasma $1 \mathrm{C}$ metabolite concentrations, analysed by liquid chromatography coupled with mass spectrometry, and cardiometabolic parameters were measured at baseline and the 3- and 6-month follow-ups.

Results Regardless of the intervention group, choline was elevated after 3 months, while cysteine and methionine remained elevated after 6 months (mixed model time effects, $p<0.05$ ). Elevated dimethylglycine and lower betaine concentrations were correlated with an unfavourable cardiometabolic profile at baseline (spearman correlations, $p<0.05$ ). However, increasing choline and dimethylglycine concentrations were associated with improvements in lipid metabolism in those receiving supplementation (regression model interaction, $p<0.05$ ).

Conclusion Choline metabolites, including choline, betaine and dimethylglycine, were central to the co-regulation of 1C metabolism and cardiometabolic health in older adults. Metabolites that indicate upregulated betaine-dependent homocysteine remethylation were elevated in those with the greatest cardiometabolic risk at baseline, but associated with improvements in lipid parameters following resistance training with nutritional supplementation. The relevance of how $1 \mathrm{C}$ metabolite status might be optimised to protect against cardiometabolic dysregulation requires further attention.
\end{abstract}

Keywords Choline $\cdot$ Homocysteine $\cdot$ Nutritional supplement $\cdot$ Older adults $\cdot$ One-carbon metabolism

\section{Introduction}

Metabolic dysregulation, leading to cardiovascular diseases, diabetes and cognitive decline are major causes of morbidity and mortality in the ageing population [1,2]. One central metabolic pathway impaired with ageing is that of onecarbon (1C) metabolism [3, 4]. Traditionally identified on the basis of elevated homocysteine (Hcy), 1C metabolism

Karl-Heinz Wagner

karl-heinz.wagner@univie.ac.at

Extended author information available on the last page of the article is central to aspects of both cognitive $[5,6]$ and cardiometabolic health $[7,8]$.

Hcy is at a critical branch point in 1C metabolism, with circulating concentrations dependent upon competing actions of the $1 \mathrm{C}$ cycle. Hcy is synthesised from the conversion of methionine to $S$-adenosylmethionine (SAM) and $S$-adenosylhomocysteine (SAH), and is remethylated through either folate and vitamin $\mathrm{B}_{12}$ or betaine-dependent pathways to support the methionine cycle, or is removed through the vitamin $\mathrm{B}_{6}$-dependent transsulfuration pathway to form cysteine [9] (Fig. 1). There is a shift towards analysing a more comprehensive $1 \mathrm{C}$ metabolite profile to provide greater insights into the coordination of circulating Hcy. 
There is substantive evidence that supports the association of $1 \mathrm{C}$ metabolites beyond Hcy with cardiometabolic health. Cysteine is proposed as an obesogenic amino acid [10] and elevated glycine conversely with a favourable cardiometabolic profile [11]. Of particular interest is choline and its downstream metabolites, betaine and dimethylglycine (DMG). These metabolites reflect Hcy remethylation by betaine-homocysteine methyltransferase (BHMT). Choline plays a critical role in lipid metabolism, as phosphatidylcholine is required for very low-density lipoprotein synthesis and hepatic lipid transport. Evidence supports divergent associations between choline and betaine and markers of cardiometabolic health. Elevated choline concentrations are typically associated with an unfavourable metabolic profile, and conversely, elevated betaine with a more protective profile for indices of anthropometry, lipid metabolism, and insulin sensitivity [12-15].

Those in their oldest years of life represent a vulnerable group, with an increased risk of dysregulated $1 \mathrm{C}$ metabolism [4] and cardiometabolic disease markers [2], yet the relationship between these processes is not well understood. Although the pathways of $1 \mathrm{C}$ metabolism are tightly interconnected, there is little evidence from studies that consider metabolites beyond distinct, targeted 1C metabolites (e.g. Hcy or choline and betaine alone). Furthermore, nutritional supplementation becomes more frequent with advancing age to support dietary adequacy and counteract age-related functional decline [16, 17]. Resistance training (RT) is another important lifestyle intervention particularly in elderly subjects, shown to improve cardiometabolic parameters [18] and 1C metabolism with evidence of circulating Hcy reduction $[19,20]$, and favourably impact related health outcomes such as cognition [21, 22]. Given the overlap of 1C metabolism and cardiometabolic markers in multiple related health outcomes, there is potential to provide various health benefits to older adults. However, the impact of RT and nutritional supplementation containing B vitamins on profiles of $1 \mathrm{C}$ metabolites and cardiometabolic parameters requires further investigation in an elderly population.

Thus, in this secondary analysis of the Vienna Active Ageing Study, we undertook a targeted approach to better understand the relationship between $1 \mathrm{C}$ metabolism and cardiometabolic health in the elderly. First, by exploring 6-month shifts in $1 \mathrm{C}$ metabolites and cardiometabolic markers, and their correlation, in the context of lifestyle interventions including RT with or without nutritional supplementation (containing protein and micronutrients, including folic acid, vitamins $B_{6}$ and $B_{12}$ ). Second, by investigating the cross-sectional association between markers of cardiometabolic health and 1C metabolites and the influence of B vitamin status.

\section{Methodology}

\section{Study design}

The present article reports on data from the Vienna Active Ageing Study [23], which uses a randomised, controlled, observer-blind design. Participants were randomly assigned to three parallel intervention groups-RT, RT with nutritional supplementation (RTS), or cognitive training (CT), which acted as the control group. Blood samples and anthropometric measurements were taken at baseline, and after 3 and 6 months of intervention. Informed written consent was obtained from all participants before they were enrolled in the trial following the Declaration of Helsinki. The Vienna Active Ageing Study was approved by the ethics committee of the City of Vienna (EK-11-151-0811) and registered at ClinicalTrials.gov, NCT01775111. This article reports on secondary exploratory outcomes; the primary outcome of the Vienna Active Ageing Study was to evaluate the effect of RT or RTS on functional parameters, including markers of chromosomal damage [24], oxidative stress [25], and DNA damage [26]. The secondary outcomes reported in this analysis include the assessment of plasma 1C metabolite concentrations and markers of cardiometabolic health.

\section{Subjects}

Over six months, 117 institutionalised older men and women (aged 65-98 years) were recruited to participate in the Vienna Active Ageing Study from five senior residences in the area of Vienna (Curatorship of the Viennese Retirement Homes). At the six-month follow-up, 95 participants were included for analysis in the current study, and a detailed participant flow diagram describing the loss of participants during follow-up has previously been reported [23]. Participants were eligible to participate if they were mentally (Mini Mental State Examination $\geq 23$ ) [27] and physically (Short Physical Performance Battery $>4$ ) stable. Inclusion and exclusion criteria have previously been described in detail [23]. Briefly, participants were sedentary $(<1 \mathrm{~h}$ of physical activity or exercise per week), aged $\geq 65$ years, and referring to the recommendations of the American Heart Association [28], were free of diseases that pose contraindication to medical training therapy, including serious cardiovascular diseases, diabetic retinopathy, and regular use of cortisonecontaining drugs. Subjects were excluded if they performed regular resistance training ( $>$ once/week in the last six months before inclusion) or were using dietary supplements and were not willing to abstain during the intervention. 


\section{Study intervention}

\section{Resistance training}

Groups receiving the training-based intervention (RT and RTS) performed two sessions of RT per week. The training protocol was developed in line with the guidelines of the American College of Sports Medicine for RT with older adults [29]. Exercise sessions were conducted on non-consecutive days and supervised by a sport scientist who recorded attendance. The training program has been described in detail by Oesen et al. [23]. Briefly, each session lasted approximately one hour, consisting of an initial ten-minute warm-up, a 30-40 min strength training using exercise bands and a chair, and a ten-minute cooldown, and the intensity and volume of the exercise were progressively increased.

\section{Resistance training with nutritional supplementation}

Participants in the RTS group followed the same training protocol as those in the RT group and additionally consumed a nutritional supplement every morning as well as directly after the bi-weekly training session. The nutritional supplement was a single $150 \mathrm{ml}$ serve of FortiFit (Nutricia, Vienna, Austria), a supplement which is freely available to purchase and designed to prevent sarcopenia in older adults. Of note, each $150 \mathrm{kcal}$ serve of this supplement provided $200 \mu \mathrm{g}$ of folic acid, $3.0 \mu \mathrm{g}$ of vitamin $\mathrm{B}_{12}, 750 \mu \mathrm{g}$ of vitamin $\mathrm{B}_{6}$, $55 \mathrm{mg}$ of choline, and $20.7 \mathrm{~g}$ of protein. Complete details of the composition of this nutritional supplement can be found in Supplementary Table 1. Participants were instructed to consume the supplement at breakfast each morning.

\section{Cognitive training}

The CT group served as the control group to minimise the bias of being part of a social group activity, as those in the RT and RTS groups received twice a week during training sessions. Those in the CT group performed coordinative or cognitive tasks [30], which were mainly memory training and finger dexterity exercises in a sitting position.

Participants in all intervention groups were instructed to maintain regular food intake, which was checked with $24 \mathrm{~h}$ diet recalls when blood samples were taken at three- and six-month follow-ups.

\section{Cardiometabolic parameters}

Waist and hip circumference, body mass and body height were measured following standardised anthropometric procedures. Body mass was measured to the nearest $0.1 \mathrm{~kg}$ (BWB 700, Tanita, Amsterdam, Netherlands) and height was measured to the nearest $0.5 \mathrm{~cm}$ using a portable stadiometer (SECA Model 217, Seca GmbH \& Co. KG, Hamburg, Germany). Body mass index (BMI) was calculated as body mass relative to height in meters squared $\left(\mathrm{kg} / \mathrm{m}^{2}\right)$.

Lipid profile (low-density lipoprotein cholesterol (LDLC), high-density lipoprotein cholesterol (HDL-C), total/ HDL-C) and triglycerides), high-sensitive insulin, plasma glucose (FPG) and glomerular filtration rate (GFR) were analysed immediately after blood sampling at a routine laboratory (study lab GmbH, Vienna, Austria). Renal function is a determinant of circulating Hcy concentrations [31, 32], and GFR was therefore used as a confounding variable in regression models. To determine Homeostatic Model Assessment of Insulin Resistance (HOMA-IR) as a marker of insulin resistance, fasting plasma glucose $(\mathrm{mg} / \mathrm{dl})$ and insulin (IU/ml) values were converted into $\mathrm{mmol} / \mathrm{L}$ (glucose) and $\mathrm{pmol} / \mathrm{L}$ (insulin), and then entered into the validated HOMA2 calculator (HOMA2 v2.2.3 $\odot \beta$, Diabetes Trials Unit, University of Oxford).

\section{One-carbon metabolites}

Following an overnight fast, blood samples were collected into EDTA-coated vacutainers. Samples were placed on ice prior to separation of plasma for analysis [33], and then separated into aliquots and stored in Eppendorf tubes at $-80^{\circ} \mathrm{C}$ until required for analysis. Samples were thawed only immediately before analysis. Ultra-high performance liquid chromatography coupled with mass spectrometry was performed to determine plasma concentrations of 11 metabolites (betaine, choline, DMG, $S$-adenosylhomocysteine (SAH), and SAM) and amino acids (cysteine, cystathionine, glycine, Hcy, methionine, and serine) involved in the regulation of $1 \mathrm{C}$ metabolism. Product/precursor ratios were calculated to provide insight into pathway regulation for betaine/choline, and DMG/betaine, which have previously been used as an index of endogenous betaine synthesis and its use, allowing inference of betaine-dependent remethylation of Hcy [34, 35].

Samples were randomised to ensure a balance of intervention group and sex across batches. The methods have been reported in detail elsewhere[36]. Briefly, plasma samples were prepared using an automated robotic liquid handling system (Eppendorf epMotion ${ }^{\circledR}$ 5075vt, Hamburg, Germany). First, $300 \mu \mathrm{l}$ of $1 \%$ formic acid in methanol was pipetted into a 96-well IMPACT ${ }^{\circledR}$ protein precipitation plate (Phenomenex, Torrance, California, USA). Next, all standards $(100 \mu \mathrm{l})$, quality controls $(100 \mu \mathrm{l})$ and standards $(100 \mu \mathrm{l})$ were spiked with $20 \mu \mathrm{l}$ of internal standard solution, agitated for $5 \mathrm{~min}(800 \mathrm{rpm})$, then filtered into a $(2 \mathrm{~mL})$ 96-well square collection plate (Phenomenex, Torrance, 
California, USA) by applying a vacuum (450 mbar). $100 \mu 1$ of Tris (2-carboxyethyl) phosphine was then dispensed into each well to allow for the separate quantification of Hcy and cysteine.

Three sets of quality control samples were included to assess recovery of standards and reproducibility of samples. Metabolites were considered acceptable if standard recoveries were between 80 and $120 \%$, and coefficients of variance were below 20\%. Cystathionine and SAH did not satisfy these requirements and were excluded from further analysis, as were SAM samples from one of three batches (34\%, $n=76$ ). For samples where a peak was not detected, a missing value was calculated as half of the minimum value of each batch [37]. A missing value was not calculated for the SAM samples that were excluded from the analysis.

\section{B vitamin status}

Plasma vitamin $\mathrm{B}_{12}$ and erythrocyte folate status were analysed using a radioimmunoassay technique, according to Müllner et al. [38]. Standard curves were drawn and sample values calculated according to the protocol provided by the kit producer (MP Biomedicals, Germany). Coefficient of variance was calculated as $5.5 \%$ for folate and $5.6 \%$ for vitamin $\mathrm{B}_{12}$. Inadequate plasma vitamin $\mathrm{B}_{12}$ and erythrocyte folate status was defined as $<150 \mathrm{pmol} / \mathrm{L}$ and $<340 \mathrm{nmol} / \mathrm{L}$, respectively, according to recommendations by the World Health Organization [39, 40].

\section{Dietary intake}

Dietary intake was assessed by interview-based $24 \mathrm{~h}$ recalls, which were performed at baseline and after 6 months. The evaluation of the records was performed using the nutritional software NUT.S (Dato Denkwerkzeuge, Vienna, Austria), which is based on the German Food Composition Database Version II.3 (Berlin, Germany) but was adapted for Austrian eating habits through the addition of typical Austrian recipes.

\section{Statistical analysis}

Before analysis, normality was assessed graphically. Due to a skewed distribution, HOMA-IR, DMG and the DMG/ betaine ratio were log-transformed for analysis. Non-transformed values were used to construct tables and graphs of summary statistics. All statistical analyses were performed using R 3.6.3 statistical software [41]. Unless otherwise specified, Alpha was set at $p \leq 0.05$, and data presented as mean (standard deviation).

Baseline differences in markers of metabolic health and $B$ vitamin status between males and females were assessed by Students $t$ test, and 1C metabolites by linear mixed models to correct for batch effect as a random factor. Baseline correlations between markers of metabolic health and 1C metabolites were assessed by Spearman rank correlation. Changes in markers of metabolic health and 1C metabolites were analysed by linear mixed models, where subject and batch effect (1C metabolites only) were fit as a random factor, and time and intervention were fit as fixed factors, including an interaction term between time and intervention. For significant interaction terms, the Sidak adjustment was applied to correct for multiple comparisons. To evaluate associations between shifts in $1 \mathrm{C}$ metabolites and markers of metabolic health, and the effect of the intervention received on this, multiple regression modelling was performed. Change (baseline-follow-up) in each metabolic marker was set as the dependent variable, and change in each metabolite was set as the independent variable in the following models. Model 1-unadjusted; Model 2-adjusted (age, sex, GFR, baseline metabolite status, and BMI for nonanthropometric-dependent variables); Model 3-adjusted model including an interaction term between the independent variable (change in metabolite concentration) and the intervention group.

\section{Results}

\section{Participant characteristics}

This secondary analysis of the Vienna Active Ageing Study included 95 participants at baseline, of which 67 had data available for $1 \mathrm{C}$ metabolites and cardiometabolic parameters at the six-month follow-up. Participants had an average age of $83( \pm 6)$ years and were predominantly female $(88.4 \%)$, which reflects the gender distribution of this age group near their statistical life expectancy in the houses of the Curatorship of Viennese Retirement homes (Table 1).

\section{Differences in B vitamins, one-carbon metabolites and cardiometabolic parameters according to sex.}

At baseline, females had a higher BMI $(p=0.045)$, but lower waist/hip ratio $(p \leq 0.001)$ than their male counterparts, with a trend towards higher serum triglycerides $(p=0.051)$ and HOMA-IR, or greater insulin resistance, $(p=0.064)$. Minor differences were seen between 1C metabolite status at baseline; females had higher plasma glycine concentrations at baseline $(p=0.003)$, and a trend towards higher plasma serine $(p=0.050)$ and lower methionine $(p=0.061)$ concentrations. No difference in B vitamin status (plasma vitamin $B_{12}$ and erythrocyte folate) or adequacy was found between males and females. Notably, there was a high prevalence of folate inadequacy in the 
total population $(93 \%$ with concentrations $<340 \mathrm{nmol} / \mathrm{L}$, the adequacy threshold recommended by the World Health Organization [39]) (Table 1).

\section{Baseline correlations between one-carbon metabolites and cardiometabolic parameters}

According to Spearman correlation analyses, cysteine concentration was positively correlated with BMI $(\rho=0.202)$ and the waist/hip ratio $(\rho=0.223)$. The waist/hip ratio was also positively correlated with both methionine $(\rho=0.219)$ and glycine $(\rho=0.398)$. HDL-C was positively correlated with glycine $(\rho=0.345)$, and the total/HDL-C ratio with DMG $(\rho=0.208)$ and DMG/betaine $(\rho=0.230)$. Choline metabolites were also correlated with triglycerides, with an inverse correlation between both betaine $(\rho=-0.300)$ and betaine/choline $(\rho=-0.287)$, while a positive correlation was found with both DMG $(\rho=0.233)$ and DMG/betaine $(\rho=0.335)$. An inverse correlation with betaine $(\rho=-0.254)$ and positive correlation with DMG/betaine $(\rho=0.207)$ was also found for HOMA-IR, but FPG was not correlated with 1C metabolites (Table 2).

\section{Changes in one-carbon metabolites and cardiometabolic parameters according to intervention group}

Across all intervention groups, an increase of the waist/ hip ratio (time effect, $p=0.008$ ) and LDL-C (time effect, $p=0.028$ ) was found at the six-month follow-up, while a decrease in HOMA-IR was found for the three-month followup, but was not sustained at the 6-month follow-up (time effect, $p \leq 0.001$ ). In the control group, we also found a reduction in FPG at the three-month follow-up, but again was not sustained at the 6-month follow-up (interaction, $p=0.006$ ). Plasma choline concentrations increased at the 3-month follow-up only (time effect, $p=0.013$ ), while Hcy, methionine and cysteine concentrations remained elevated at the six-month follow-up (time effect, $p \leq 0.001$ ). An interaction effect was found for serine $(p=0.024)$. However, post hoc comparisons were not significant upon applying the Sidak correction (Table 3, Fig. 1). Changes in B vitamin concentrations from baseline to follow-up have been presented elsewhere [24]. Of relevance to the present findings, an increase in erythrocyte folate and plasma vitamin $\mathrm{B}_{12}$ concentrations was reported to increase in the RTS group only [24].

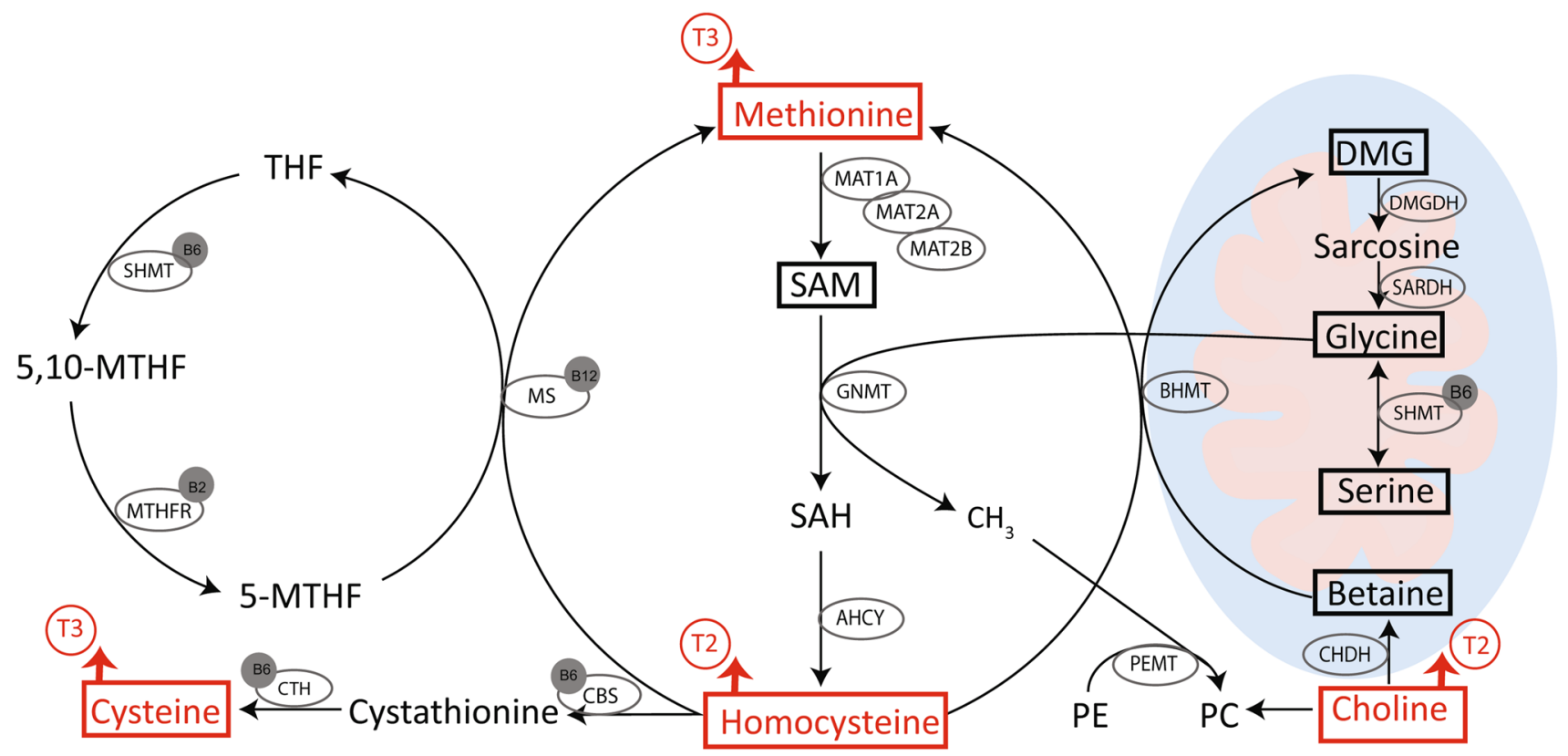

Fig. 1 Overview of one-carbon metabolism, and the impact of intervention with resistance training (RT), resistance training with supplementation (RTS), or cognitive training (CT) for 6 months on onecarbon metabolites. Metabolites analysed in this paper are highlighted in bold and outlined in a box, B vitamins acting as co-enzymes are in a grey circle, and enzymes are outlined in an oval. A red arrow indicates a change from baseline to follow-up ( $\uparrow$, increase; $\downarrow$, decrease) and time point (T2, 3-month; T3, 6-month) for metabolites where a significant time effect $(p<0.05)$ was found in linear mixed models. No changes according to the intervention group were found for any metabolites (interaction, $p>0.05$ ). $A H C Y S$-adenosylhomocysteine hydrolase, $B H M T$ betaine-homocysteine methyltransferase, $C B S$ cystathionine $\beta$-synthase, $C H D H$ choline dehydrogenase, $C T H$ cystathionine $\lambda$-lyase, $D M G$ dimethylglycine, $G N M T$ glycine $N$-methyltransferase, $M A T$ methionine adenosyltransferase, $M S$ methionine synthase, $P C$ phosphatidylcholine, $P E$ phosphatidylethanolamine, $P E M T$ phosphatidylethanolamine methyltransferase, $S A H S$-adenosylhomocysteine, SAM S-adenosylmethionine, SARDH sarcosine dehydrogenase, SHMT serine hydroxymethyltransferase (SHMT1, cytosolic; SHMT2, mitochondrial), THF tetrahydrofolate 
Table 1 Baseline status of one-carbon metabolites, cardiometabolic parameters and B vitamins according to sex

\begin{tabular}{|c|c|c|c|c|c|}
\hline Characteristic & Variable & $\begin{array}{l}\text { Total } \\
(n=95)\end{array}$ & $\begin{array}{l}\text { Males } \\
(n=11)\end{array}$ & $\begin{array}{l}\text { Females } \\
(n=84)\end{array}$ & $p$ value \\
\hline & Age (years) & $83.1(5.8)$ & $83.4(5.2)$ & $82.4(6.7)$ & 0.110 \\
\hline \multirow[t]{8}{*}{ Metabolic Health } & Body mass index $\left(\mathrm{kg} / \mathrm{m}^{2}\right)$ & $29.1(4.8)$ & $26.9(3.4)$ & $29.4(4.9)$ & $0.045^{*}$ \\
\hline & Waist/Hip ratio & $0.86(0.07)$ & $0.93(0.04)$ & $0.85(0.07)$ & $<0.001^{*}$ \\
\hline & Serum LDL Cholesterol (mmol/L) & $3.17(0.97)$ & $2.77(0.98)$ & $3.22(0.96)$ & 0.178 \\
\hline & Serum HDL cholesterol (mmol/L) & $1.65(0.44)$ & $1.51(0.36)$ & $1.67(0.44)$ & 0.200 \\
\hline & Serum Total/HDL-cholesterol ratio & $3.47(1.01)$ & $3.23(0.66)$ & $3.50(1.04)$ & 0.240 \\
\hline & Serum Triglycerides $(\mathrm{mmol} / \mathrm{L})$ & $1.31(0.51)$ & $1.09(0.34)$ & $1.33(0.52)$ & 0.051 \\
\hline & Plasma glucose $(\mathrm{mmol} / \mathrm{L})$ & $5.71(1.10)$ & $5.43(0.84)$ & $5.74(1.13)$ & 0.280 \\
\hline & HOMA-IR & $2.41(2.30)$ & $1.65(1.18)$ & $2.51(2.39)$ & 0.064 \\
\hline \multirow[t]{11}{*}{ One-carbon metabolites } & Betaine $(\mu \mathrm{M})$ & $30.8(9.36)$ & $35.0(10.5)$ & $30.3(9.13)$ & 0.193 \\
\hline & Choline $(\mu \mathrm{M})$ & $14.3(2.59)$ & $14.4(3.49)$ & $14.3(2.48)$ & 0.477 \\
\hline & Cysteine $(\mu \mathrm{M})$ & $103(21.9)$ & $104(13.6)$ & $102(22.8)$ & 0.539 \\
\hline & $\mathrm{DMG}(\mu \mathrm{M})$ & $2.84(1.26)$ & $2.74(1.25)$ & $2.85(1.27)$ & 0.749 \\
\hline & Glycine $(\mu \mathrm{M})$ & $195(48.1)$ & $157(25.6)$ & $200(48.2)$ & $0.003^{*}$ \\
\hline & Homocysteine $(\mu \mathrm{M})$ & $2.20(1.07)$ & $2.00(1.12)$ & $2.23(1.07)$ & 0.943 \\
\hline & Methionine $(\mu \mathrm{M})$ & $22.45(3.19)$ & $24.3(3.21)$ & $22.2(3.12)$ & 0.061 \\
\hline & $S$-adenosylmethionine (nM) & $56.6(16.8)$ & $53.3(22.0)$ & $57.1(16.1)$ & 0.555 \\
\hline & Serine $(\mu \mathrm{M})$ & $95.8(26.6)$ & $79.9(16.1)$ & $97.9(27.0)$ & 0.050 \\
\hline & Betaine/choline & $2.20(0.69)$ & $2.44(0.46)$ & $2.16(0.71)$ & 0.211 \\
\hline & DMG/betaine & $0.10(0.05)$ & $0.09(0.06)$ & $0.10(0.05)$ & 0.940 \\
\hline \multirow[t]{4}{*}{ B vitamin status } & Plasma Vitamin $\mathrm{B}_{12}(\mathrm{pmol} / \mathrm{L})$ & $380(333)$ & $460(410)$ & $370(324)$ & 0.456 \\
\hline & Prevalence of vitamin $B_{12}$ inadequacy ${ }^{a}$ & $21 \%$ & $20 \%$ & $30 \%$ & 0.479 \\
\hline & Erythrocyte folate $(\mathrm{nmol} / \mathrm{L})$ & $181(107)$ & $185(95.4)$ & $181(109)$ & 0.962 \\
\hline & Prevalence of folate inadequacy ${ }^{b}$ & $93 \%$ & $94 \%$ & $89 \%$ & 0.589 \\
\hline \multirow[t]{10}{*}{ Dietary intake } & Energy (kcal) & $1556(354)$ & $1705(298)$ & $1544(357)$ & 0.176 \\
\hline & Protein (\% energy intake) & 16.2 & 17.3 & 16.1 & \\
\hline & Fat (\% energy intake) & 35.0 & 34.4 & 35.1 & \\
\hline & Carbohydrate (\% energy intake) & 48.8 & 48.3 & 48.8 & \\
\hline & Fibre $(\mathrm{g})$ & $21.7(30.6)$ & $21.7(7.0)$ & $21.7(31.8)$ & 0.176 \\
\hline & Protein (g/kg/body weight) & $0.83(0.30)$ & $0.85(0.24)$ & $0.83(0.27)$ & 0.701 \\
\hline & Folate $(\mu \mathrm{g})$ & $261(124)$ & $300(121)$ & $257(125)$ & 0.450 \\
\hline & Riboflavin (mg) & $1.17(0.41)$ & $1.02(0.35)$ & $1.18(0.42)$ & 0.347 \\
\hline & Vitamin $\mathrm{B}_{6}(\mathrm{mg})$ & $1.56(0.78)$ & $1.73(0.47)$ & $1.55(0.80)$ & 0.205 \\
\hline & Vitamin $B_{12}(\mu \mathrm{g})$ & $5.05(3.03)$ & $5.59(3.64)$ & $5.01(2.99)$ & 0.618 \\
\hline
\end{tabular}

Data presented as mean (standard deviation). All biochemical measures are fasting samples. Abbreviations: $D M G$ dimethylglycine

${ }^{a}$ Inadequate plasma vitamin $\mathrm{B}_{12}$ was defined as $<150 \mathrm{pmol} / \mathrm{L}$ according to the World Health Organization[40]

${ }^{\mathrm{b}}$ Inadequate erythrocyte folate was defined as $<340 \mathrm{nmol} / \mathrm{L}$ according to the World Health Organization[39]

*Indicates a significant difference $(p<0.05)$ between sex for variables at baseline

\section{Longitudinal associations between changes in one-carbon metabolites and cardiometabolic parameters}

Increasing Hcy concentrations were associated with a rise in the waist/hip ratio in both unadjusted $(\beta=0.020, p=0.008)$ and adjusted models $(\beta=0.020, p=0.042)$, while a positive association between shifts in cysteine concentrations and the waist/hip ratio was significant in an unadjusted model only $\left(\beta=8.2^{-4}, p=0.035\right)$. Increasing DMG concentrations were associated with a decline of BMI $(\beta=-0.410, p=0.009)$ and LDL-C $(\beta=-10.8, p=0.031)$, although only that with BMI remained significant when adjusted for confounding variables $(\beta=-0.400, p=0.038)$. Increasing DMG was also associated with a rise in HOMA-IR in an adjusted model only $(\beta=0.72, p=0.018)$. A positive association was also found between shifts in the ratio of total/HDL-C and DMG/betaine, a marker of upregulated betaine-dependent 
Table 2 Baseline correlation analysis between one-carbon metabolites and cardiometabolic parameters

BMI Waist/hip ratio LDL-Cholesterol HDL-Cholesterol Total/HDL- Triglycerides Glucose HOMA-IR
Cholesterol

\begin{tabular}{lllllllll}
\hline Betaine & $-0.191^{*}$ & $0.180^{*}$ & 0.076 & $0.196^{*}$ & -0.116 & $-0.300^{* *}$ & -0.086 & $-0.254^{* *}$ \\
Choline & -0.135 & 0.050 & 0.031 & 0.032 & -0.035 & -0.053 & $-0.201^{*}$ & -0.104 \\
Cysteine & $0.202^{* *}$ & $0.223^{* *}$ & -0.094 & -0.167 & 0.061 & 0.158 & 0.053 & 0.091 \\
DMG & 0.043 & -0.022 & 0.122 & -0.083 & $0.208^{* *}$ & $0.233^{* *}$ & -0.182 & 0.077 \\
Glycine & 0.071 & -0.398 & 0.044 & $0.345^{* * *}$ & $-0.173^{*}$ & -0.128 & -0.125 & -0.063 \\
Homocysteine & 0.170 & 0.065 & -0.080 & -0.145 & 0.045 & 0.097 & 0.101 & 0.080 \\
Methionine & -0.046 & $0.219 * *$ & -0.099 & -0.004 & -0.127 & $-0.199 *$ & 0.039 & -0.088 \\
S-adenosylmethionine & 0.143 & 0.134 & -0.032 & -0.238 & 0.206 & -0.150 & 0.083 & 0.142 \\
Serine & 0.109 & -0.088 & 0.078 & 0.074 & 0.004 & -0.049 & -0.092 & -0.046 \\
Betaine/choline & -0.100 & 0.146 & -0.019 & $0.180^{*}$ & -0.147 & $-0.287 * *$ & 0.043 & -0.176 \\
DMG/Betaine & 0.144 & -0.057 & 0.081 & -0.160 & $0.230^{* *}$ & $0.335^{* * *}$ & -0.068 & $0.207 * *$ \\
\hline
\end{tabular}

Correlation coefficients presented according to Spearman correlation analysis. ${ }^{*} p<0.100, * * p<0.05$, *** $p<0.01$. Abbreviations: $D M G$ dimethylglycine, SAM $S$-adenosylmethoinine

Hcy remethylation, in unadjusted $(\beta=6.17, p=0.034)$ and adjusted $(\beta=8.74, p=0.014$ ) models (Table 4 , Supplementary Table 2).

The most consistent effect of the intervention was seen in markers of lipid metabolism. In the RTS group, increasing concentrations of choline, cysteine, DMG, glycine, and serine were associated with a decline in the ratio of total cholesterol/HDL-C compared to the CT and RT groups. Similarly, increasing concentrations of choline, cysteine, DMG, and methionine were associated with reduced triglyceride concentrations (interaction, $p<0.05$ ) in the RTS group compared to the CT and RT groups. Rising betaine and serine concentrations were also associated with a decline in HOMA-IR in the RTS group compared to the CT and RT groups, while positive associations between shifts in methionine and cysteine with HOMA-IR were found in both the RTS and RT groups compared to those in the CT group (Fig. 2). Full details of these effects can be found in Supplementary Table 2.

\section{Discussion}

This study is a novel investigation into the impact of lifestyle interventions on a comprehensive profile of 1C metabolites and cardiometabolic parameters in an elderly population (65-98 years). Metabolites reflecting upregulated betainedependent Hcy remethylation were associated with an unfavourable lipid profile at baseline. However, increasing DMG, cysteine, and choline concentrations were associated with improved lipid parameters in those receiving nutritional supplementation, which likely reflects underlying shifts in methylation status and choline availability, both of which play a critical role in lipid metabolism. This study highlights the complex relationship between $1 \mathrm{C}$ metabolism and cardiometabolic health, which choline metabolites appear central to. Notably, our findings highlight that the relationship between 1C metabolites and cardiometabolic health may be modified by resistance training combined with nutritional supplementation. These findings bolster the relevance of 1C metabolites in healthy ageing, and further research is required to understand whether optimising $1 \mathrm{C}$ metabolite status might confer cardiometabolic and related benefits in very old adults.

Hcy is an established risk factor for cardiovascular disease [7]. Despite growing interest in the association between Hcy and the metabolic syndrome, this has yielded inconsistent findings and is poorly characterised [8, 42-46]. While associations between Hcy and cardiometabolic markers were limited, metabolites reflective of betaine-dependent Hcy remethylation were more closely associated with parameters of glucose and lipid metabolism. Our findings indicate that upregulation of the BHMT pathway to support Hcy remethylation is associated with an unfavourable cardiometabolic profile; betaine and the ratio of betaine/choline were inversely correlated with HOMA-IR and triglycerides, while DMG and the ratio of DMG/betaine were positively correlated with triglycerides, total/HDL-C and HOMA-IR. Shifts in DMG concentrations were associated with changes in cardiometabolic markers, yet the direction of response was inconsistent, as increasing DMG concentrations were associated with improvements in LDL-C and BMI, but worsening insulin sensitivity in adjusted models. These findings in part support previous reports of betaine being associated with a more favourable cardiometabolic profile, although based on the available evidence, we would have expected to find a more divergent association between betaine and choline with these metabolic markers rather than between betaine 


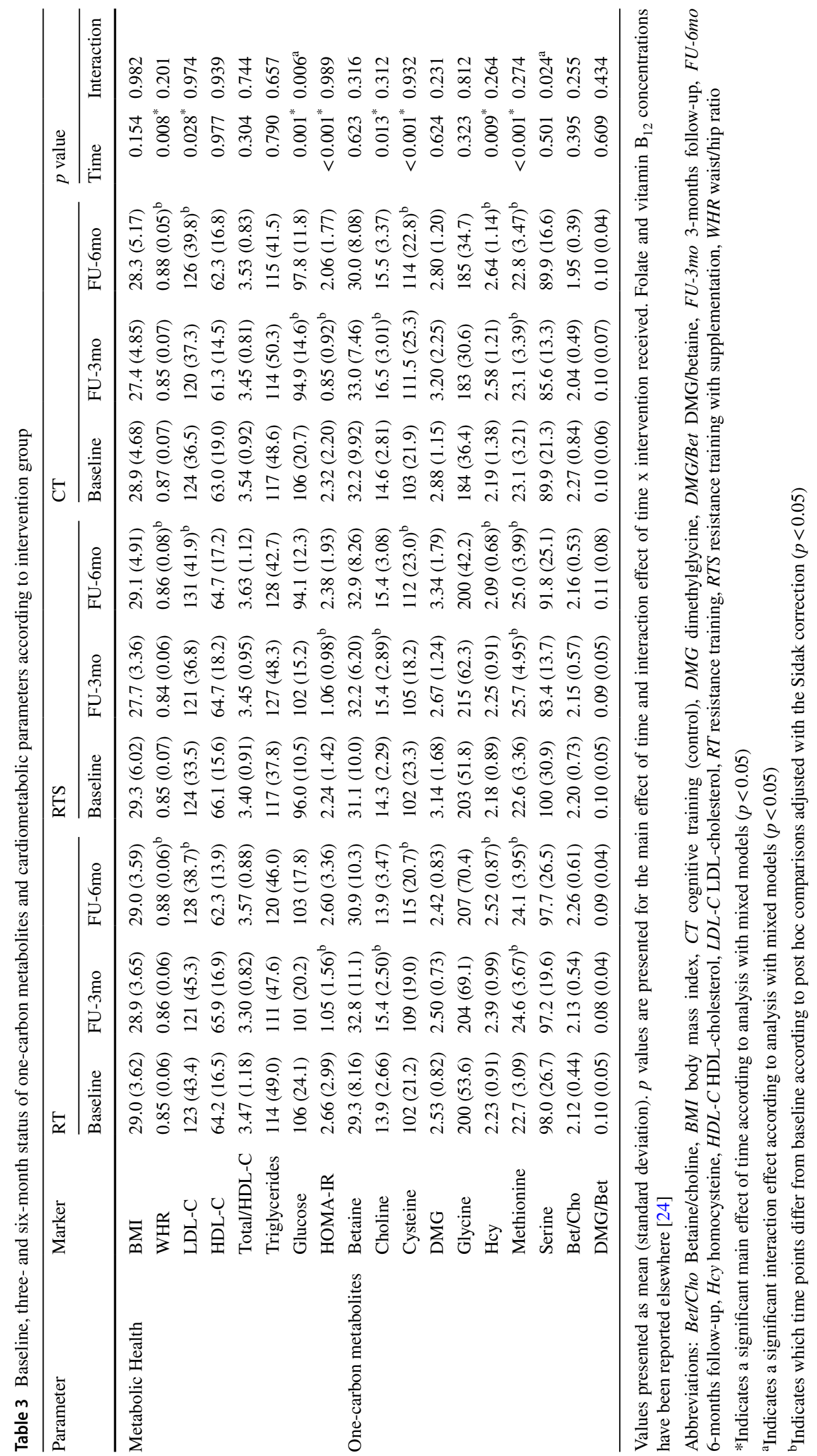




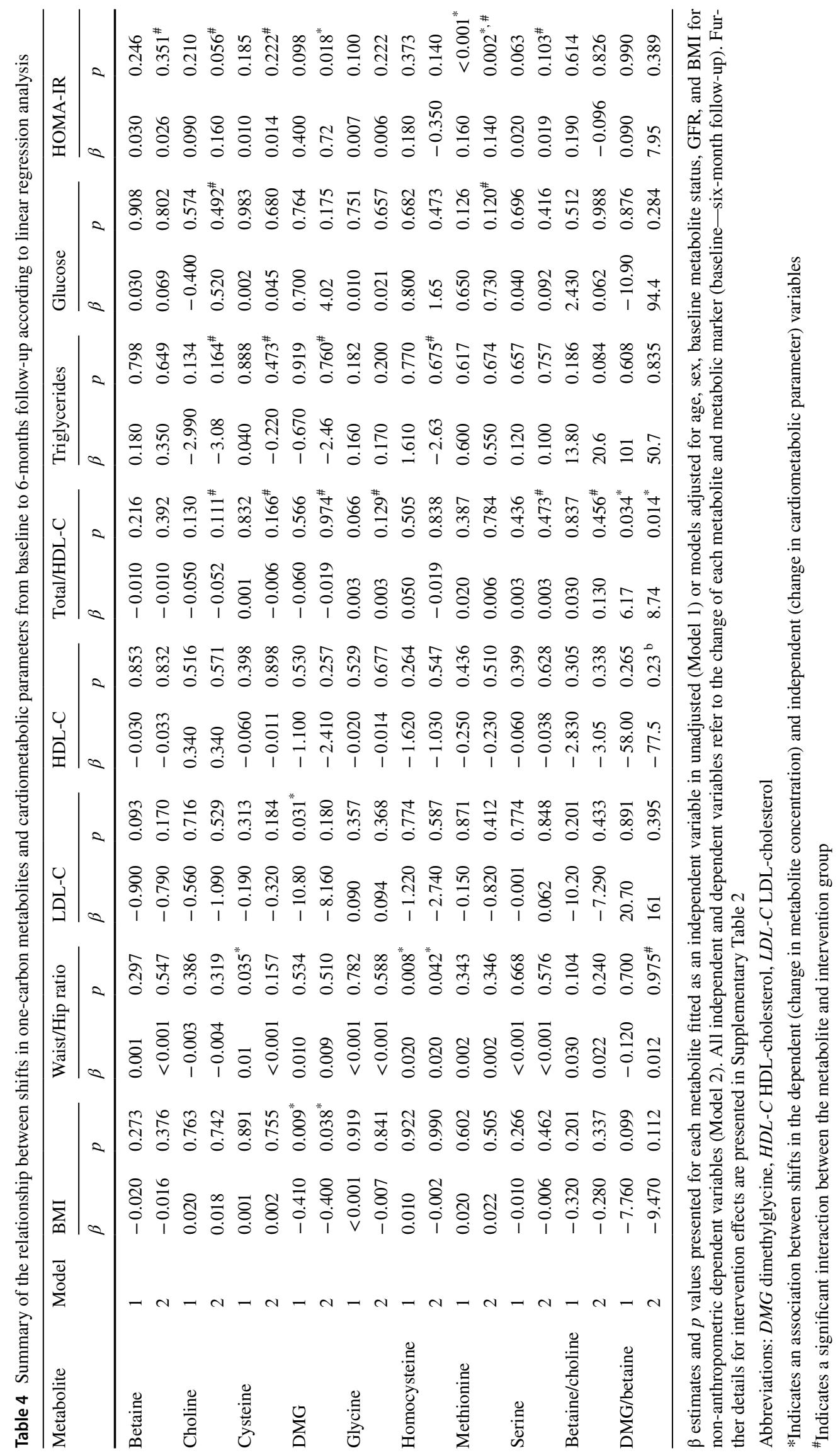


Fig. 2 Effect of intervention with resistance training (RT), resistance training with supplementation (RTS), or cognitive training (CT) on associations between six-month shifts in one-carbon metabolites and cardiometabolic parameters. Scatter plots depict associations between changes in choline concentrations with a total/HDL-cholesterol and b triglyceride concentrations; cysteine concentrations with c total/HDL-cholesterol and d triglyceride concentrations, and dimethylglycine concentrations with e total/HDL-cholesterol and $\mathbf{f}$ triglyceride concentrations. Data are based on linear regression analyses presented in Table 4 and Supplementary Table 2, and refer to a model adjusted for confounding variables (age, sex, glomerular filtration rate, baseline metabolite concentrations, and BMI), and includes an interaction term between the intervention group and the dependent variable. Change (baseline-follow-up) in metabolites are set as dependent variables, and change in cardiometabolic markers are set as independent variables
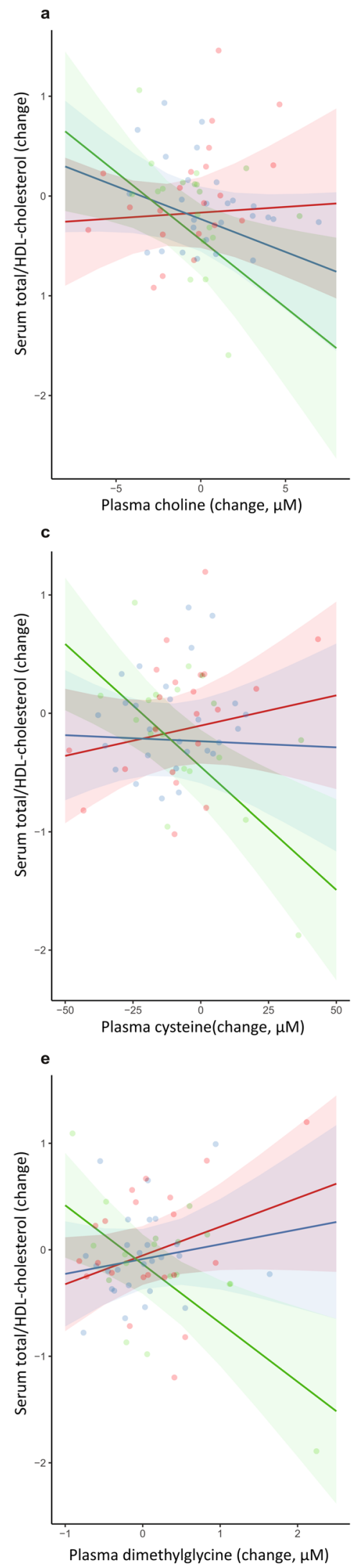
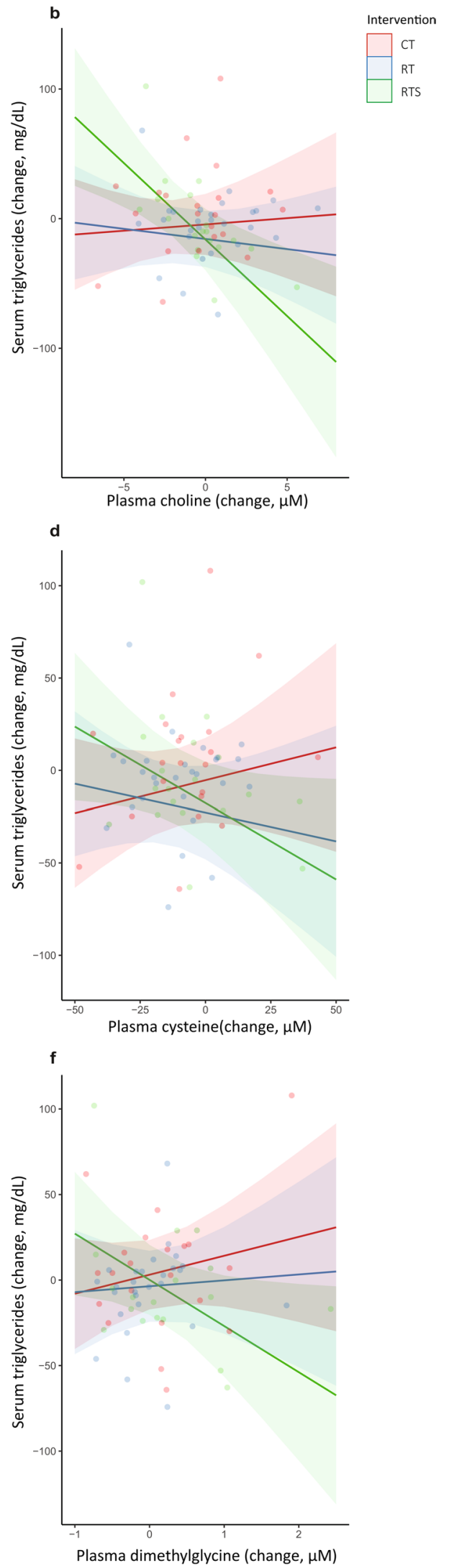
and DMG [12-15]. There is a relative paucity of literature regarding DMG, although lower concentrations have previously been associated with higher blood glucose, increased insulin resistance, and an increased risk of incident diabetes [47]. The only known pathway for DMG synthesis results from betaine donating a methyl group for the remethylation of Hcy, and thus is considered to reflect BHMT activity [48, 49]. Upregulation of this pathway is evidently implicated in numerous aspects of cardiometabolic risk, which, for the most part, appears to be an unfavourable relationship.

Interestingly, supplementation modified the longitudinal relationship between shifts in these markers of Hcy remethylation and lipid metabolism. Increasing DMG, choline, and cysteine concentrations were associated with improvements in the lipid profile only in those receiving supplementation. Although we are unable to provide further insight into mechanisms, this is plausibly mediated by shifts in methylation status and choline availability for the synthesis of phosphatidylcholine. With increased flux through BHMT, phosphatidylcholine synthesis from choline is restricted, as there is greater demand for choline oxidation to support betaine availability. In turn, this places an increased demand on the synthesis of phosphatidylcholine from phosphatidylethanolamine, which requires labile methyl groups [50]. Indeed, betaine supplementation has been shown to increase hepatic phosphatidylcholine concentrations and the ratio of phosphatidylcholine/phosphatidylethanolamine [51]. In another study, betaine supplementation improved whole-body glucose homeostasis and energy expenditure [52]. Supplementation with krill oil has been shown to reduce triglycerides, which was suggested to be through increased phosphatidylcholine intake $(1750 \mathrm{mg} /$ day $)$ and choline availability leading to enhanced processes dependent on methyl donors, including flux over BHMT [53]. The Fortifit supplement used in the current study contains only a small amount of choline $(55 \mathrm{mg})$. Although B vitamin supplementation may increase choline status [54], greater supplemental concentrations, or a longer follow-up period may have been required to see similar improvements in the lipid profile of our participants.

While increasing cysteine concentrations were associated with favourable changes in lipid parameters in those receiving supplementation, this should be considered alongside the relationship between cysteine and body composition. Cysteine concentrations were correlated with higher BMI and waist/hip ratio at baseline. Increasing cysteine concentrations were also associated with a rise in waist/hip ratio during the six-month follow-up in simple regression analyses regardless of the intervention received, but not when adjusted for confounding variables. These findings in part align with those from the Hordaland Homocysteine study $[55,56]$, where cysteine was positively associated with $\mathrm{BMI}$ and fat mass in both cross-sectional and longitudinal analyses in a large cohort of middle-aged and older adults [10]. Beyond cysteine's link to methylation status, mechanisms have been extensively reviewed and proposed by Elshorbagy et al. [10]. Our findings support the role of cysteine in body composition, but require careful interpretation in elderly populations, as there is a shift upwards in the BMI range that is considered healthy and protective against mortality in older adults [57]. Vitamin $\mathrm{B}_{6}$ is required for cysteine synthesis (through cystathionine- $\beta$-synthase and cystathionine- $\lambda$-lyase), yet vitamin $B_{6}$ deficiency is prevalent amongst elderly populations $[58,59]$. The balance is complex, and further studies are required to better understand the mechanisms underlying the role of cysteine in the regulation of body composition and lipid metabolism, particularly in vulnerable populations that require special consideration, such as those in their oldest years.

It is interesting to note that Hcy did not decline in those receiving the RT or RTS intervention, an observation previously been reported with both B vitamin supplementation $[60,61]$ and RT $[19,20]$ in older adults. This might be explained by the nutritional supplement used here, which contained a moderate amount of $200 \mu \mathrm{g}$ folic acid per serve compared to $400 \mu \mathrm{g}$ shown to reduce Hcy in other elderly populations $[60,61]$. The Fortifit supplement also provided $20.7 \mathrm{~g}$ of protein per serve, thus increasing the supply of dietary methionine. Further, participants in the current study were older (mean, $83 \pm 6$ years) than other elderly populations who have shown a decline in Hcy with RT interventions (range 60-80 years) [19, 20]. However, caution should be given to the interpretation of these findings given the analytical technique used. While the mass spectrometry technique used has been peer-reviewed with good internal reproducibility [36], the concentrations of Hcy are below expected reference ranges [63] which limits the clinical interpretation of our results. The discrepancy in Hcy concentrations can be explained by a lower concentration of Tris (2-carboxyethyl) phosphine used, an agent used in sample preparation to reduce disulfide bonds in cystine and homocystine to allow quantification of cysteine and Hcy, compared to other studies [64].

This study provides valuable insight into the response of $1 \mathrm{C}$ metabolism to intervention those in their oldest years of life. A key strength of our study lies in the elderly population included ( $83 \pm 6$ years). Octogenarians and nonagenarians are the fastest growing proportion of our ageing population [65], yet are under-represented in research on 1C metabolism. Lifestyle interventions, such as supplementation and exercise, are promoted to optimise health in this vulnerable population, yet to our knowledge, this is the first study to investigate the effect of such interventions on cardiometabolic parameters in advanced age. These results, of course, should not be generalised to early agers where B vitamin status might be expected to be more 
adequate than in our cohort. Further, while the mass spectrometry technique used here gave a comprehensive profile of plasma $1 \mathrm{C}$ metabolites, but does not quantify sarcosine, trimethylamine $N$-oxide, or other choline species (e.g. phosphatidylcholine). The inclusion of these metabolites would help to interpret the findings in the current study, as would quantifying urinary 1C metabolites [66].

\section{Conclusion}

This study highlights the complex relationship between 1C metabolites and cardiometabolic health in an elderly institutionalised population, which choline metabolites were central to. While RT or RTS did not improve plasma 1C metabolites or cardiometabolic risk profiles after six months, more subtle changes in pathway regulation were indicated. 1C metabolites reflecting upregulated betainedependent Hcy remethylation were correlated with an unfavourable cardiometabolic profile. However, the direction of association between these $1 \mathrm{C}$ metabolites and the lipid profile was modified in those receiving RT with nutritional supplementation. These findings highlight the potential to promote successful ageing through improving health outcomes underpinned by these interconnected pathways, however, further research is required to demonstrate the potential of optimising $1 \mathrm{C}$ metabolite status such that cardiometabolic benefit is conferred in older adults.

Supplementary Information The online version contains supplementary material available at https://doi.org/10.1007/s00394-021-02607-y.

Acknowledgements We thank the Curatorship of Viennese Retirement Homes and its residents for taking part in the study. We thank Dr Matthew Barnett for the coordination of the AgResearch Ltd Strategic Science Investment Fund. Nicola Gillies thanks Professor Clare Wall and Professor Richard Mithen for PhD supervision, and the University of Auckland and the Hope Foundation for scholarship support.

Authors' contributions Author responsibilities were as follows; Conceptualisation: BF, BH-S, BW, DC-S, K-HW, SO; Methodology: BF, BH-S, SO, BW, K-HW; Formal analysis: AT, BF, BH-S, NAG, SO, BW, K-HW; Investigation AT, BF, BH-S, BW, K-HW, MH, SO; Data curation BF, BH-S, BW, K-HW, NAG, SO; Writing-original draft: NAG; Writing — review and editing: AT, AMM, BF, BH-S, BW, DC-S, K-HW, MH, NCR, SO; Visualisation-NAG; Supervision: K-HW; Project administration: BW, K-HW Funding acquisition: KH-W, BW, DC-S, NCR. All authors approved the final version of the manuscript for submission.

Funding Open access funding provided by University of Vienna. This work was supported by the University of Vienna by funding the Research Platform Active Ageing and the EU-IRSES-318962BIOAGE project. The analysis of $1 \mathrm{C}$ metabolites was supported by AgResearch Limited through the Strategic Science Investment Fund (Contracts A19079 and A21246: Nutritional strategies for an ageing population)

\section{Declarations}

Conflict of interest The authors declare that they have no conflict of interest.

Ethics approval The Vienna Active Ageing Study was approved by the ethics committee of the City of Vienna (EK-11-151-0811) and registered at ClinicalTrials.gov, NCT01775111.

Consent to participate Informed written consent was obtained from all participants before they were enrolled in the trial following the Declaration of Helsinki.

Open Access This article is licensed under a Creative Commons Attribution 4.0 International License, which permits use, sharing, adaptation, distribution and reproduction in any medium or format, as long as you give appropriate credit to the original author(s) and the source, provide a link to the Creative Commons licence, and indicate if changes were made. The images or other third party material in this article are included in the article's Creative Commons licence, unless indicated otherwise in a credit line to the material. If material is not included in the article's Creative Commons licence and your intended use is not permitted by statutory regulation or exceeds the permitted use, you will need to obtain permission directly from the copyright holder. To view a copy of this licence, visit http://creativecommons.org/licenses/by/4.0/.

\section{References}

1. Mendis S, Puska P, Norrving B (2011) Global atlas on cardiovascular disease prevention and control. World Heal Organ

2. Dominguez LJ, Barbagallo M (2007) The cardiometabolic syndrome and sarcopenic obesity in older persons. J Cardiometab Syndr 2:183-189. https://doi.org/10.1111/j.1559-4564.2007. 06673.x

3. Selhub J, Jacques PF, Rush D et al (1993) Vitamin status and intake as primary determinants of homocysteinemia in an elderly population. JAMA 270:2693-2698. https://doi.org/10.1001/jama. 1993.03510220049033

4. Porter K, Hoey L, Hughes CF et al (2016) Causes, consequences and public health implications of low B-vitamin status in ageing. Nutrients 8:725. https://doi.org/10.3390/nu8110725

5. Smith A, Refsum H (2016) Homocysteine, B Vitamins, and cognitive impairment. Annu Rev Nutr 36:211-239. https://doi.org/10. 1146/annurev-nutr-071715-050947

6. Mathers JC (2015) Impact of nutrition on the ageing process. $\mathrm{Br}$ J Nutr 113:S18-S22. https://doi.org/10.1017/S00071145140032 37

7. Homocysteine Studies Collaboration (2002) Homocysteine and risk of ischemic heart disease and stroke: a meta-analysis. JAMA 288:2015-2022. https://doi.org/10.1001/jama.288.16.2015

8. Hajer GR, Van Der Graaf Y, Olijhoek JK et al (2007) Levels of homocysteine are increased in metabolic syndrome patients but are not associated with an increased cardiovascular risk, in contrast to patients without the metabolic syndrome. Heart 93:216220. https://doi.org/10.1136/hrt.2006.093971

9. Selhub J (2002) Folate, vitamin B12 and vitamin B6 and one carbon metabolism. J Nutr Health Aging 6:39-42

10. Elshorbagy A, Kozich V, Smith A, Refsum H (2012) Cysteine and obesity: Consistency of the evidence across epidemiologic, animal and cellular studies. Curr Opin Clin Nutr Metab Care 15:49-57 
11. Alves A, Bassot A, Bulteau AL et al (2019) Glycine metabolism and its alterations in obesity and metabolic diseases. Nutrients 11:1356

12. Lever M, George PM, Atkinson W et al (2011) Plasma lipids and betaine are related in an acute coronary syndrome cohort. PLoS ONE 6:e21666

13. Roe AJ, Zhang S, Bhadelia RA et al (2017) Choline and its metabolites are differently associated with cardiometabolic risk factors, history of cardiovascular disease, and MRI-documented cerebrovascular disease in older adults. Am J Clin Nutr 105:1283-1290. https://doi.org/10.3945/ajcn.116.137158

14. Konstantinova SV, Tell GS, Vollset SE et al (2008) Divergent associations of plasma choline and betaine with components of metabolic syndrome in middle age and elderly men and women. J Nutr 138:914-920

15. Cheng S, Rhee EP, Larson MG et al (2012) Metabolite profiling identifies pathways associated with metabolic risk in humans. Circulation 125:2222-2231. https://doi.org/10.1161/CIRCULATIO NAHA.111.067827

16. Qato DM, Wilder J, Schumm LP et al (2016) Changes in prescription and over-the-counter medication and dietary supplement use among older adults in the United States, 2005 vs 2011. JAMA Intern Med 176:473-482

17. Gahche JJ, Bailey RL, Potischman N, Dwyer JT (2017) Dietary supplement use was very high among older adults in the United States in 2011-2014. J Nutr 147:1968-1976

18. Strasser B, Siebert U, Schobersberger W (2010) Resistance training in the treatment of the metabolic syndrome: a systematic review and meta-analysis of the effect of resistance training on metabolic clustering in patients with abnormal glucose metabolism. Sport Med 40:397-415

19. Vincent KR, Braith RW, Bottiglieri T et al (2003) Homocysteine, and lipoprotein levels following resistance training in older adults. Prev Cardiol 6:197-203

20. Vincent HK, Bourguignon C, Vincent KR (2006) Resistance training lowers exercise-induced oxidative stress and homocysteine levels in overweight and obese older adults. Obesity 14:1921-1930

21. Cassilhas RC, Viana VAR, Grassmann V et al (2007) The impact of resistance exercise on the cognitive function of the elderly. Med Sci Sports Exerc 39:1401

22. Liu-Ambrose T, Donaldson MG (2009) Exercise and cognition in older adults: Is there a role for resistance training programmes? Br J Sports Med 43:25-27

23. Oesen S, Halper B, Hofmann M et al (2015) Effects of elastic band resistance training and nutritional supplementation on physical performance of institutionalised elderly - a randomized controlled trial. Exp Gerontol 72:99-108

24. Franzke B, Halper B, Hofmann M et al (2015) The effect of six months of elastic band resistance training, nutritional supplementation or cognitive training on chromosomal damage in institutionalized elderly. Exp Gerontol 65:16-22

25. Franzke B, Schober-Halper B, Hofmann M et al (2018) Age and the effect of exercise, nutrition and cognitive training on oxidative stress- the Vienna Active Aging Study (VAAS), a randomized controlled trial. Free Radic Biol Med 121:69-77

26. Franzke B, Halper B, Hofmann M et al (2015) The impact of six months strength training, nutritional supplementation or cognitive training on DNA damage in institutionalised elderly. Mutagenesis 30:147-153

27. Folstein MF, Folstein SE, McHugh PR (1975) "Mini-mental state". A practical method for grading the cognitive state of patients for the clinician. J Psychiatr Res 12:189-198. https://doi. org/10.1016/0022-3956(75)90026-6

28. Williams MA, Haskell WL, Ades PA et al (2007) Resistance exercise in individuals with and without cardiovascular disease: 2007 update: a scientific statement from the American Heart Association Council on clinical cardiology and council on nutrition, physical activity, and metabolism. Circulation 116:572-584

29. Nelson ME, Rejeski WJ, Blair SN et al (2007) Physical activity and public health in older adults: recommendation from the American College of Sports Medicine and the American Heart Association. Circulation 116:1094

30. Gatterer G, Croy A (2004) Mental fitness in aging. Springer, Vienna

31. Bostom AG, Lathrop L (1997) Hyperhomocysteinemia in endstage renal disease: prevalence, etiology, and potential relationship to arteriosclerotic outcomes. Kidney Int 52:10-20

32. Arnadottir M, Hultberg B, Nilsson-Ehle P, Thysell H (1996) The effect of reduced glomerular filtration rate on plasma total homocysteine concentration. Scand J Clin Lab Invest 56:41-46

33. Ueland PM, Refsum H, Stabler SP et al (1993) Total homocysteine in plasma or serum: methods and clinical applications. Clin Chem 39:1764-1779

34. Yan J, Winter LB, Burns-Whitmore B et al (2012) Plasma choline metabolites associate with metabolic stress among young overweight men in a genotype-specific manner. Nutr Diabetes 2:e49. https://doi.org/10.1038/nutd.2012.23

35. Bae S, Ulrich CM, Neuhouser ML et al (2014) Plasma choline metabolites and colorectal cancer risk in the women's health initiative observational study. Cancer Res 74:7442-7452. https://doi. org/10.1158/0008-5472.CAN-14-1835

36. Andraos S, Goy M, Albert BB et al (2020) Robotic automation of a UHPLC/MS-MS method profiling one-carbon metabolites, amino acids, and precursors in plasma. Anal Biochem 592:113558. https://doi.org/10.1016/j.ab.2019.113558

37. Wei R, Wang J, Su M et al (2018) Missing value imputation approach for mass spectrometry-based metabolomics data. Sci Rep 8:1. https://doi.org/10.1038/s41598-017-19120-0

38. Müllner E, Brath H, Toferer D et al (2013) Genome damage in peripheral blood lymphocytes of diabetic and non-diabetic individuals after intervention with vegetables and plant oil. Mutagenesis 28:205-211

39. WHO (2015) Serum and red blood cell folate concentrations for assessing folate status in populations. Vitamin and Mineral Nutrition Information System. Geneva: World Health Organization. http://apps.who.int/iris/bitstream/10665/162114/1/WHO_NMH_ NHD_EPG_15.01.pdf?ua $=1$

40. de Benoist B (2008) Conclusions of a WHO Technical Consultation on folate and vitamin B12 deficiencies. Food Nutr Bull 29:S238-S244. https://doi.org/10.1177/15648265080292S129

41. R Core Team (2017) R: A language and environment for statistical computing. R Core Team

42. Catena C, Colussi G, Nait F et al (2015) Elevated homocysteine levels are associated with the metabolic syndrome and cardiovascular events in hypertensive patients. Am J Hypertens 28:943-950

43. Wang X, Ye P, Cao R et al (2014) The association of homocysteine with metabolic syndrome in a community-dwelling population: homocysteine might be concomitant with metabolic syndrome. PLoS ONE 9:e113148

44. Garcin JM, Cremades S, Garcia-Hejl C et al (2006) Is hyperhomocysteinemia an additional risk factor of the metabolic syndrome? Metab Syndr Relat Disord 4(3): 185

45. Rhee EJ, Hwang ST, Lee WY et al (2007) Relationship between metabolic syndrome categorized by newly recommended by international diabetes federation criteria with plasma homocysteine concentration. Endocr J 54:995-1002. https://doi.org/10.1507/ endocrj.K07E-018

46. Björck J, Hellgren M, Råstam L, Lindblad U (2006) Associations between serum insulin and homocysteine in a Swedish populationa potential link between the metabolic syndrome and hyperhomocysteinemia: the Skaraborg project. Metabolism 55:1007-1013 
47. Magnusson M, Wang TJ, Clish C et al (2015) Dimethylglycine deficiency and the development of diabetes. Diabetes 64:3010 3016. https://doi.org/10.2337/db14-1863

48. Allen RH, Stabler SP, Lindenbaum J (1993) Serum betaine, $N$, $\mathrm{N}$-dimethylglycine and $\mathrm{N}$-methylglycine levels in patients with cobalamin and folate deficiency and related inborn errors of metabolism. Metabolism 42(11):1448-1460

49. Schwab U, Alfthan G, Aro A, Uusitupa M (2011) Long-term effect of betaine on risk factors associated with the metabolic syndrome in healthy subjects. Eur J Clin Nutr 65:70-76. https://doi.org/10. 1038/ejen.2010.230

50. Jacobs RL, Stead LM, Devlin C et al (2005) Physiological regulation of phospholipid methylation alters plasma homocysteine in mice. J Biol Chem 280:28299-28305. https://doi.org/10.1074/jbc. M501971200

51. Deminice R, Da Silva RP, Lamarre SG et al (2015) Betaine supplementation prevents fatty liver induced by a high-fat diet: effects on one-carbon metabolism. Amino Acids 47:839-846. https://doi. org/10.1007/s00726-014-1913-x

52. Ejaz A, Martinez-Guino L, Goldfine AB et al (2016) Dietary betaine supplementation increases Fgf21 levels to improve glucose homeostasis and reduce hepatic lipid accumulation in mice. Diabetes 65:902-912. https://doi.org/10.2337/db15-1094

53. Bjørndal B, Bruheim I, Lysne V et al (2018) Plasma choline, homocysteine and vitamin status in healthy adults supplemented with krill oil: a pilot study. Scand J Clin Lab Invest 78:527-532. https://doi.org/10.1080/00365513.2018.1512716

54. van Wijk N, Watkins CJ, Böhlke M et al (2012) Plasma choline concentration varies with different dietary levels of vitamins B6, B12 and folic acid in rats maintained on choline-adequate diets. Br J Nutr 107:1408-1412. https://doi.org/10.1017/S000711451 1004570

55. El-Khairy L, Vollset SE, Refsum H, Ueland PM (2003) Predictors of change in plasma total cysteine: longitudinal findings from the Hordaland Homocysteine Study. Clin Chem 49:113-120. https:// doi.org/10.1373/49.1.113

56. Elshorbagy AK, Nurk E, Gjesdal CG et al (2008) Homocysteine, cysteine, and body composition in the Hordaland Homocysteine Study: Does cysteine link amino acid and lipid metabolism? Am J Clin Nutr 88:738-746
57. Johnson MA, Bales CW (2014) Is there a best body mass index for older adults? Moving closer to evidence-based recommendations regarding "overweight", health, and mortality. J Nutr Gerontol Geriatr 33:1-9

58. Bates CJ, Pentieva KD, Prentice A et al (1999) Plasma pyridoxal phosphate and pyridoxic acid and their relationship to plasma homocysteine in a representative sample of British men and women aged 65 years and over. Br J Nutr 81:191-201. https:// doi.org/10.1017/S0007114599000380

59. Morris MS, Picciano MF, Jacques PF, Selhub J (2008) Plasma pyridoxal 5'-phosphate in the US population: the National Health and Nutrition Examination Survey, 2003-2004. Am J Clin Nutr 87:1446-1454

60. Brönstrup A, Hages M, Pietrzik K (1999) Lowering of homocysteine concentrations in elderly men and women. Int J Vitam Nutr Res 69:187-193. https://doi.org/10.1024/0300-9831.69.3.187

61. McKay DL, Perrone G, Rasmussen H et al (2000) Multivitamin/ mineral supplementation improves plasma B-vitamin status and homocysteine concentration in healthy older adults consuming a folate-fortified diet. J Nutr 130:3090-3096. https://doi.org/10. 1093/jn/130.12.3090

62. Silva ADSE, Da Mota MPG (2014) Effects of physical activity and training programs on plasma homocysteine levels: a systematic review. Amino Acids 46:1795-1804

63. Nygard O, Refsum H, Ueland PM, Vollset SE (1998) Major lifestyle determinants of plasma total homocysteine distribution: the Hordaland Homocysteine Study. Am J Clin Nutr 67:263-270. https://doi.org/10.1093/ajen/67.2.263

64. Ueland PM, Midttun $\varnothing$, Windelberg A et al (2007) Quantitative profiling of folate and one-carbon metabolism in large-scale epidemiological studies by mass spectrometry. Clin Chem Lab Med 45:1737-1745. https://doi.org/10.1515/CCLM.2007.339

65. United Nations Department of Economic and Social Affairs (2019) World population ageing 2019: highlights. United Nations Department of Economic and Social Affairs

66. Svingen GFT, Schartum-Hansen H, Pedersen ER et al (2016) Prospective associations of systemic and urinary choline metabolites with incident type 2 diabetes. Clin Chem 62:755-765. https://doi. org/10.1373/clinchem.2015.250761

\section{Authors and Affiliations}

\section{Nicola A. Gillies ${ }^{1,2} \cdot$ Bernhard Franzke $^{3} \cdot$ Barbara Wessner $^{3,4} \cdot$ Barbara Schober-Halper $^{3} \cdot$ Marlene Hofmann $^{3}$. Stefan Oesen ${ }^{3} \cdot$ Anela Tosevska $^{3,5} \cdot$ Eva-Maria Strasser $^{6} \cdot$ Nicole C. Roy $^{1,2,7,8,9} \cdot$ Amber M. Milan $^{1,7,8}$. David Cameron-Smith ${ }^{1,2,10} \cdot$ Karl-Heinz Wagner $^{3,11}$ [0]}

1 Liggins Institute, University of Auckland, Auckland, New Zealand

2 Riddet Institute, Palmerston North, New Zealand

3 Research Platform Active Ageing, University of Vienna, Althanstrasse 14, 1090 Vienna, Austria

4 Department of Sports Medicine, Exercise Physiology and Prevention, University of Vienna, Vienna, Austria

5 Internal Medicine III, Division of Rheumatology, Medical University of Vienna, Vienna, Austria

6 Institute for Physical Medicine and Rehabilitation, Kaiser Franz Josef Hospital - Social Medical Center South, Vienna, Austria
7 Food, Nutrition and Health, AgResearch, Hamilton, New Zealand

8 The High-Value Nutrition National Science Challenge, Auckland, New Zealand

9 Department of Human Nutrition, University of Otago, Dunedin, New Zealand

10 Singapore Institute for Clinical Sciences, Agency for Science, Technology, and Research, Singapore, Singapore

11 Department of Nutritional Sciences, University of Vienna, Vienna, Austria 